\title{
On Generalisation of Dual-Thermocouple Sensor Characterisation to RTDs
}

\author{
Colin Brown ${ }^{\# 1}$, Robert J. Kee ${ }^{\# 2}$, George Irwin ${ }^{\# 3}$ Seán F. McLoone ${ }^{* 4}$, Peter C. Hung ${ }^{* 5}$ \\ \# Virtual Engineering Centre, Queen's University Belfast, \\ Belfast BT9 $5 H N$, Northern Ireland \\ ${ }^{1}$ cbrown17@qub.ac.uk, ${ }^{2}$ r.kee@qub.ac.uk, ${ }^{3}$ g.irwin@qub.ac.uk \\ ${ }^{*}$ Department of Electronic Engineering, National University of Ireland Maynooth, \\ Maynooth, Co. Kildare \\ ${ }^{4}$ sean.mcloone@eeng.nuim.ie, ${ }^{5}$ phung@eeng.nuim.ie
}

\begin{abstract}
Intrusive temperature sensors such as thermocouples and resistance temperature detectors (RTDs) have become industry standards for simple and cost-effective temperature measurement. However, many situations require the use of physically robust and therefore low bandwidth temperature sensors. Much work has been published on dual-thermocouple thermometry as a means of obtaining increased sensor bandwidth from relatively robust thermocouples, which are assumed to have firstorder response. This contribution seeks to determine if RTDs, which are known to have approximately first-order response [1], can also be characterised using the dual-thermocouple approach. Experimental results show that the response of an RTD cannot be represented by a first-order model with sufficient accuracy to allow successful application of this method. Furthermore, simulation studies demonstrated that if a sensor exhibits even marginally second-order response, highly inaccurate temperature reconstructions follow. It is concluded that a higher-order model that more accurately reflects RTD response would be required for successful dual-RTD characterisation.
\end{abstract}

\section{INTRODUCTION}

Instantaneous in situ measurement of rapidly varying temperatures is often a requirement in diverse areas of research and industry and is required to gain an understanding of many dynamic systems. However, achieving such measurements in an accurate, reliable and cost-effective manner is a challenging problem. Although non-intrusive acoustic and laser-based temperature measurement techniques are capable of providing virtually instantaneous temperature measurements, their use is restricted to very specialised applications due to their complexity and high cost. As a result, comparatively routine, convenient and less expensive intrusive temperature sensors are commonly utilised. The two most common intrusive temperature sensing technologies in use today are thermocouples and RTDs and between them, they fulfil most temperature sensing requirements.

Intrusive sensors provide an indication of the temperature at their measurement point. However, this is not necessarily the true temperature of the surrounding gas, as the sensor has a finite heat capacity and the heat transfer to it is governed by the laws of conduction, convection and radiation. In an automotive exhaust pipe, for example, the exhaust pipe wall and the thermocouple mount are generally cooler than the exhaust gas. Therefore, heat will be conducted to the mount and radiated to the exhaust wall, causing the temperature of the sensor to deviate from the true gas temperature. This temperature difference is said to comprise conduction and radiation errors and these exist in both transient and steadystate situations. During transient temperature measurement, the bandwidth of the sensor becomes an important factor. The bandwidth of a thermocouple $\left(\omega_{B}\right)$, for example, is dependent on its diameter according to Eq. (1), where $d$ is the diameter of the thermocouple wire, $v$ is the velocity of the gas, $k$ is almost invariant and $m$ is a constant, typically within the range $0.3 \leqslant m \leqslant 0.7[2],[3]$.

$$
\omega_{B}=k d^{m-2} v^{m}
$$

Thus, as thermocouple diameters decrease, so too do their time constants and the range of temperature variation frequencies that can then be measured without significant attenuation and phase shifting also increases. This increased bandwidth is however achieved at the expense of durability and ease of manufacture. This effect is not exclusive to thermocouples, but affects all intrusive temperature sensors. The design of such a sensor therefore represents a compromise between accuracy, robustness, low cost and rapidity of response. Harsh environments such as automotive exhaust systems require the use of robust sensors, resulting in low bandwidths. There are numerous similar applications, where the measured temperature has significantly faster dynamics than the sensor. Dynamic compensation of the measured signal is therefore necessary if the true gas temperature is to be reconstructed.

Much research has been published on increasing the effective bandwidth of thermocouples using software-based compensation techniques. However, these rely on having an accurate model of the sensor response. According to conservation of energy, the convective thermocouple heat transfer is equal to the sum of its thermal inertia plus its conductive and radiative heat transfers. For a well designed thermocouple with long fine wires, both the conductive and radiative heat transfer terms become negligible when compared to the convective heat transfer to the thermocouple from the surrounding airflow 
[2]-[4]. Thermocouple response can then be represented by a simplified first-order lag system [3], [5], [6] with time constant $\tau$, as in Eq. (2), where $T_{g}$ is the true gas temperature and $T_{m}$ is the measured temperature.

$$
T_{g}(t)=T_{m}(t)+\tau \dot{T}_{m}(t) .
$$

If $\tau$ is known for a particular thermocouple, then this model can be used to take into account the thermal transient state of the sensor. The measured temperature and its derivative can then be used to reconstruct the true gas temperature. Since $\tau$ is a function of the bandwidth $\omega_{B}\left(\tau=2 \pi \omega_{B}^{-1}\right)$, Eq. (1) shows that it varies with gas velocity and is unknown a priori. Furthermore, noise corrupted measurements make accurate computation of $\dot{T}_{m}(t)$ in Eq. (2) impractical. A single thermocouple will therefore not provide sufficient information for accurate in situ characterisation.

This problem has led to the development of alternative approaches, one of which involves the use of dual-thermocouple sensors with dissimilar time constants, allied with reconstruction algorithms. Numerous articles on time constant estimation and subsequent temperature reconstructions on this basis have been published e.g. [2]-[4]. However, these techniques require specific and detailed a priori knowledge and/or rely on restrictive assumptions. In addition, they are generally subject to singularities and are sensitive to noise. Hung et al. [7] later proposed an approach based on discrete-time system identification that assumes nothing about the sensor time constants, yet is superior to previous methods in terms of time constant estimation accuracy and noise tolerance.

Although much research has been published on dualthermocouple sensors and their characterisation, no similar work exists for RTDs, the most commonly used temperature sensor in mass production vehicles. For dual-thermocouple characterisation, it is assumed that the sensor exhibits firstorder response. The motivation of this study is to determine if RTDs can be characterised by a first-order model with sufficient accuracy to allow application of existing dualthermocouple characterisation techniques to a dual-RTD probe in order that the application domain of the technique can be broadened. Specifically, the dual-sensor probe was constructed using the general industry standard for RTDs, the Pt-100 and the discrete-time system identification technique described by Hung et al. [7] was used to determine the parameters necessary for sensor characterisation.

The remainder of the paper is organised as follows. Section II gives an overview of the dual-thermocouple method. System indentification based dual-thermocouple characterisation is introduced in Section III. Experimental and simulation results are then presented in Section IV. Finally, Section V provides a summary and overall conclusions.

\section{Dual-Thermocouple Characterisation}

Although many variations on dual-thermocouple thermometry have recently been proposed, they are all based on the principles first published by Pfriem [8]. The technique assumes that two thermocouples of unequal diameter are set at a distance that ensures they are exposed to identical measurement environments, most importantly in terms of the gas temperature $T_{g}$ and gas velocity $v$. Recalling that a thermocouple can accurately be modelled using a first-order model (Eq. (2)), the first-order models given by Eqs. (3) and (4) then follow, where the subscripts 1 and 2 denote two thermocouples, the diameters of which are $d_{1}$ and $d_{2}$ respectively.

$$
\begin{aligned}
& T_{g}(t)=T_{m 1}(t)+\tau_{1} \dot{T}_{m 1}(t) \\
& T_{g}(t)=T_{m 2}(t)+\tau_{2} \dot{T}_{m 2}(t)
\end{aligned}
$$

From (1), it can be shown that the ratio of the time constants of two such thermocouples is a function of thermocouple geometry alone and is therefore approximately invariant [9]:

$$
\alpha=\frac{\tau_{1}}{\tau_{2}}=\frac{\omega_{B 2}}{\omega_{B 1}}=\frac{k d_{2}^{m-2} v^{m}}{k d_{1}^{m-2} v^{m}}=\left(\frac{d_{1}}{d_{2}}\right)^{2-m} .
$$

Combining Eqs. 3, 4 and 5 to give Eq. 6 and assuming knowledge of $\alpha, T_{g}(t)$ can be estimated directly using Eq. 7 .

$$
\begin{gathered}
\frac{T_{g}(t)-T_{m 1}(t)}{T_{g}(t)-T_{m 2}(t)}=\alpha \frac{\dot{T}_{m 1}(t)}{\dot{T}_{m 2}(t)} \\
T_{g}(t)=\frac{T_{m 1}(t) \dot{T}_{m 2}(t)-\alpha \dot{T}_{m 1}(t) T_{m 2}(t)}{\dot{T}_{m 2}(t)-\alpha \dot{T}_{m 1}(t)}
\end{gathered}
$$

Despite the relative simplicity of this method, numerical difficulties occur when attempting to reconstruct $T_{g}$ from noisy measurements. These are primarily due to the requirement for instantaneous derivative values and a possible singularity due to the form of the denominator. Tagawa and Ohta [2] avoided the singularity problems of Eq. (7) by minimising the timeaveraged, mean-square difference between the reconstructions from Eqs. (3) and (4), thus allowing $\tau_{1}$ and $\tau_{2}$ to be estimated. However, this method proved unreliable under noisy conditions, often producing infeasible results.

\section{SySTEM IDENTIFICATION BASED DUAL-THERMOCOUPLE CHARACTERISATION}

Hung et al. [7] proposed an approach based on discrete-time system identification that assumes no a priori knowledge of the thermocouple time constants. The discrete-time first-order difference equation, equivalent to the single thermocouple model of Eq. (2) is given by Eq. (8). Here, $a$ and $b$ are parameters and $k$ is the sample instants. Assuming zero-order-holds on the input signal and a sampling interval $\tau_{s}$, the parameters of the discrete and continuous time thermocouples are related by Eq. (9). This assumption is a valid approximation provided that the system sampling rate greatly exceeds the bandwidth of $T_{g}$.

$$
\begin{gathered}
T_{m}(k)=a T_{m}(k-1)+b T_{g}(k-1), \quad k \geq 1 \\
a=\exp \left(-\frac{\tau_{s}}{\tau}\right), \quad b=1-a
\end{gathered}
$$


Using difference equations equivalent to Eq. (8) for both thermocouples, Hung et al. [7] eliminated $T_{g}$ and introduced a parameter $\beta$, defined as $\beta \triangleq b_{2} / b_{1}$ to give Eq. (10).

$$
\Delta T_{m 2}^{k}=\beta \Delta T_{m 1}^{k}+b_{2} \Delta T_{m 12}^{k-1},
$$

where $\Delta T_{m 1}^{k}, \Delta T_{m 2}^{k}$, and $\Delta T_{m 12}^{k-1}$ are temperature differences defined as

$$
\begin{aligned}
& \Delta T_{m 1}^{k}=T_{m 1}(k)-T_{m 1}(k-1) \\
& \Delta T_{m 2}^{k}=T_{m 2}(k)-T_{m 2}(k-1) \\
& \Delta T_{m 12}^{k-1}=T_{m 1}(k-1)-T_{m 2}(k-1)
\end{aligned}
$$

If $T_{m 1}(k)$ is selected as an input and $T_{m 2}(k)$ as an output, then the synthetic ARX structure shown in Fig. 1 follows. Hung et al. [7] first proposed this model and estimated the unknown system parameters using generalised total leastsquares. The significance of this technique is that the sensor can be characterised by two measured values, without the requirement for a priori information.

$$
T_{m l}(k) \longrightarrow \beta \frac{1-\left(1-b_{1}\right) z^{-1}}{1-\left(1-\beta b_{1}\right) z^{-1}} \longrightarrow T_{m 2}(k)
$$

Fig. 1. Pseudo ARX model for dual-thermocouple characterisation.

For an $M$-sample data set Eq. (10) can be expressed in vector-matrix form as

$$
\mathbf{Y}=\mathbf{X} \theta
$$

with $\mathbf{Y}=\Delta \mathbf{T}_{m 2}^{k}, \mathbf{X}=\left[\begin{array}{cc}\Delta \mathbf{T}_{m 1}^{k} & \Delta \mathbf{T}_{m 12}^{k-1}\end{array}\right]$ and $\theta=\left[\begin{array}{ll}\beta & b_{2}\end{array}\right]^{T}$. Here $\Delta \mathbf{T}_{m 1}^{k}, \Delta \mathbf{T}_{m 2}^{k}$ and $\Delta \mathbf{T}_{m 12}^{k-1}$ are $(M-1)$-vectors. If accurate estimates of the parameters relating $T_{m 1}(k)$ to $T_{m 2}(k)$ are obtained, $\tau_{1}$ and $\tau_{2}$ follow from Eq. (9), allowing two sets of gas temperatures $T_{g 1}$ and $T_{g 2}$ to be calculated using Eqs. (3) and (4). A final reconstructed temperature, $T_{g r}$ then follows from Eq. (3) or from a weighted average of Eqs. (3) and (4) based on the time constant estimates.

\section{Results AND Simulation}

Two wire-wound Pt-100s with diameters of 1.5 and $4.5 \mathrm{~mm}$ were exposed to a step change in gas temperature under a constant gas velocity of $15 \mathrm{~m} / \mathrm{s}$. The measured output from these sensors is shown in Fig. 2 and is compared with a reference measurement $T_{m r e f}$ from a bare-wire thermocouple with a diameter of $50 \mu \mathrm{m}$. Temperature reconstructions were attempted using this data with the algorithms described in Sections II and III. Unfortunately, none of these produced useful time constant estimates, with the result that temperature reconstructions were not possible. This failure suggests that Pt-100s might not be accurately represented by a firstorder model, the underlying assumption of all the previously discussed characterisation methods.

The MATLAB ${ }^{\mathrm{TM}}$ System Identification Toolbox ${ }^{\mathrm{TM}}$ was used to calculate the first- and second-order transfer functions relating $T_{m r e f}$ to the measured output of each sensor $\left(T_{m 1}\right.$

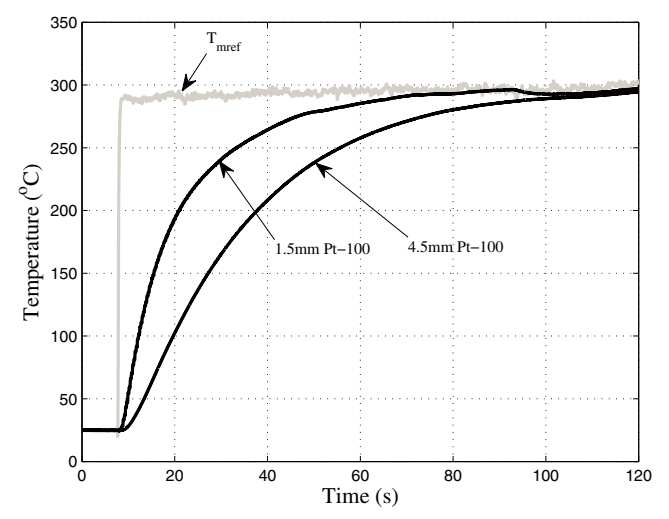

Fig. 2. Step change in gas temperature measured using wire-wound Pt-100s.

and $T_{m 2}$ ), as given in Tables I and II. Figures 3 and 4 then illustrate the output of these models when $T_{m r e f}$ is given as an input. A first-order model is seen to represent Pt-100 response reasonably accurately, while a second-order one offers only marginal reductions in root mean-square-error (RMSE).

TABLE I

DERIVED FIRST- AND SECOND-ORDER TRANSFER FUNCTIONS FOR $1.5 \mathrm{MM}$ DIAMETER WIRE-WOUND PT- 100.

\begin{tabular}{|c|c|}
\hline Model Order & Derived Transfer Function \\
\hline $1^{\text {st }}$ & $G(s)=\frac{0.99}{(1+12.48 s)}$ \\
$2^{\text {nd }}$ & $G(s)=\frac{0.99}{(1+0.17 s)(1+12.30 s)}$ \\
\hline
\end{tabular}

TABLE II

DERIVED FIRST- AND SECOND-ORDER TRANSFER FUNCTIONS FOR 4.5 MM DIAMETER WIRE-WOUND PT- 100.

\begin{tabular}{|c|c|}
\hline Model Order & Derived Transfer Function \\
\hline $1^{\text {st }}$ & $G(s)=\frac{1.03}{(1+28.95 s)}$ \\
$2^{\text {nd }}$ & $G(s)=\frac{1.01}{(1+1.08 s)(1+25.72 s)}$ \\
\hline
\end{tabular}

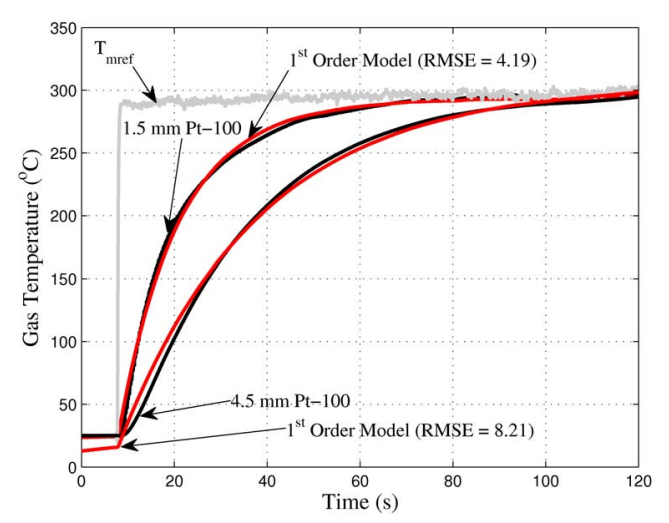

Fig. 3. Approximation of Pt-100 output using a first-order model. 


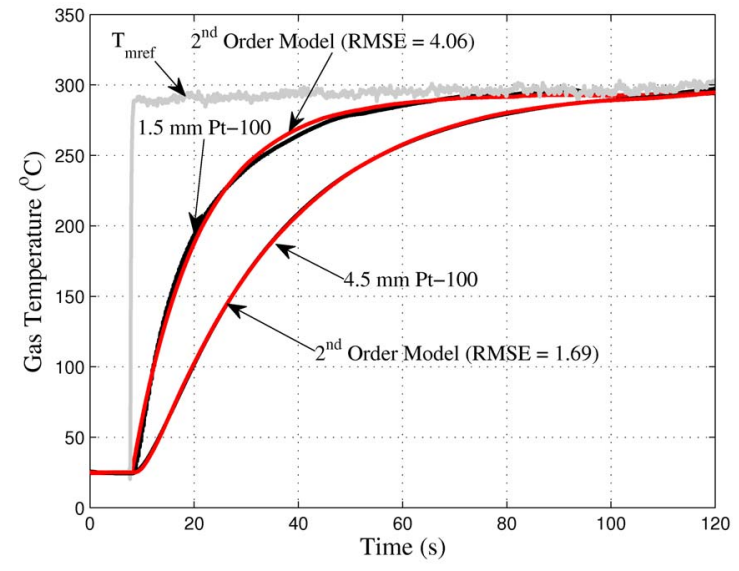

Fig. 4. Approximation of Pt-100 output using a second-order model.

To investigate the effect on algorithm performance of deviating from a first-order sensor model, the previously determined transfer functions were used to simulate the two Pt-100s when subjected to a step change in temperature. The $1.5 \mathrm{~mm}$ Pt-100 was assumed to have a first-order transfer function, while the $4.5 \mathrm{~mm}$ Pt-100 was assumed to have a second-order one (Fig. 5 ) with an additional pole at $-\frac{1}{a_{p}}$, and a gain of $a_{p}$ to ensure that its steady-state gain was maintained at unity as $a_{p}$ varied.

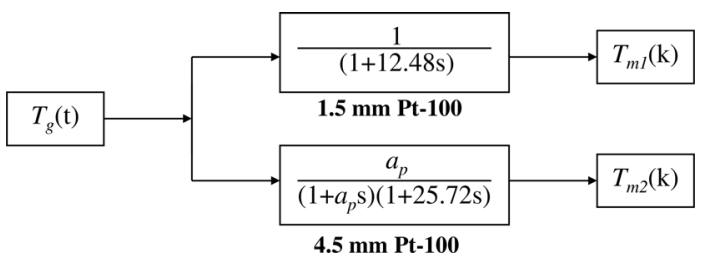

Fig. 5. Simulated Pt-100 output using first- and second-order models.

When the dominant pole is zero, the second-order transfer function for the $4.5 \mathrm{~mm}$ Pt-100 in Fig. 5 becomes a first-order one. As the pole approaches zero, the sensor response will increasingly reflect that of a first-order sensor as illustrated in Fig. 6. Therefore, by beginning with a model where $a_{p}=0$, and increasing $a_{p}$, the effect of using a sensor that increasingly deviates from the required first-order response can be examined.

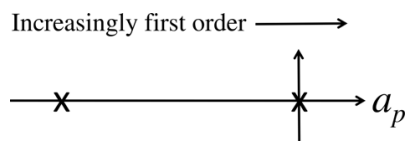

Fig. 6. Pole diagram.

A simulated step increase in temperature $T_{g}$ was applied to the models shown in Fig. 5, allowing the outputs $T_{m 1}$ and $T_{m 2}$ to be calculated. Time constants were then estimated from $T_{m 1}$ and $T_{m 2}$ using the dual-thermocouple characterisation method described by Hung et al. [7]. The true gas temperature $T_{g r}$ was then calculated using Eq. (3), allowing the RMSE between $T_{g r}$ and $T_{m r e f}$ to be calculated. This was repeated for a range of $a_{p}$ values to produce the graph shown in Fig. 7. Figure 7 demonstrates that as the additional pole becomes more dominant, i.e. the sensor response increasingly reflects that of a second-order sensor, the errors between the measured and reconstructed temperatures increase rapidly. Furthermore, apart from those at very small values of $a_{p}$, the related temperature reconstructions were very inaccurate. To put this into context, the $a_{p}$ values of both Pt-100s have been marked on the figure, showing that reconstructions using current difference equation methods with these sensors would be impossible.

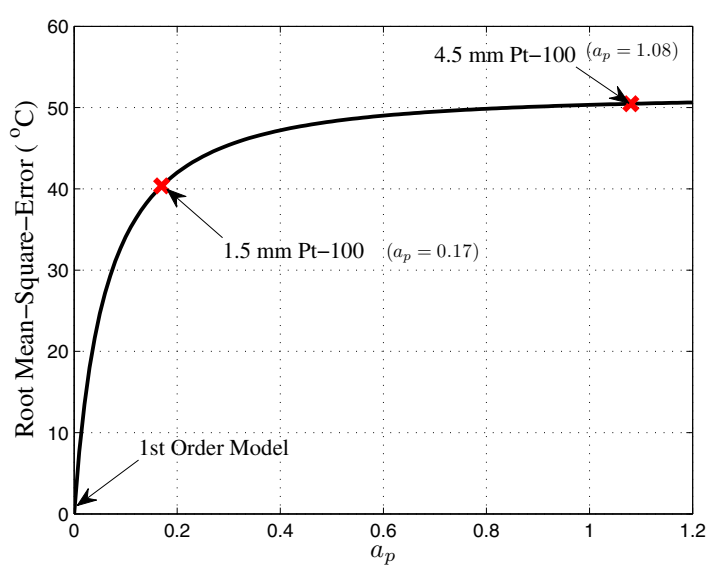

Fig. 7. Variation in mean-squared-error between $T_{g r}$ and $T_{g r e f}$ with $a_{p}$.

Reconstructed temperatures at $a_{p}=0.001$ and $a_{p}=0.1$ are then shown in Figs. 8 and 9 respectively. It is clear that the accuracy of temperature reconstruction decreases dramatically as the sensor model deviates from the assumed first-order response.

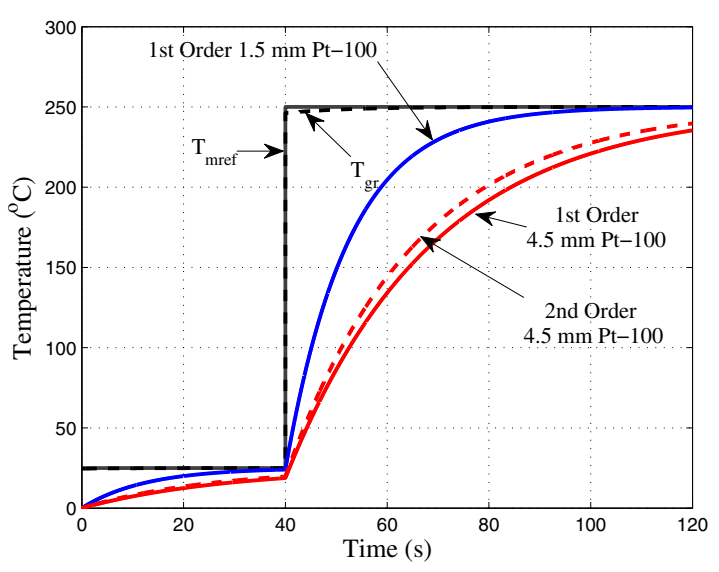

Fig. 8. Temperature reconstruction with $a_{p}=0.001$.

To more accurately reflect the response of the two Pt100 s, the previous simulation study was repeated with both sensors now assumed to be second-order as in Fig. 10. The 


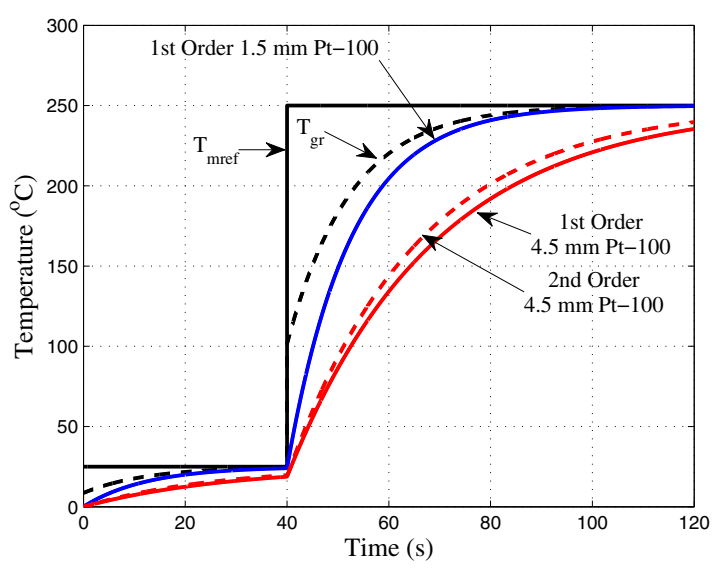

Fig. 9. Temperature reconstruction with $a_{p}=0.1$.

second-order models from Tables I and II were used, with the dominant pole of the second Pt-100 again being varied. Figure 11 shows that with this simulation, the temperature reconstruction accuracy generally improved.

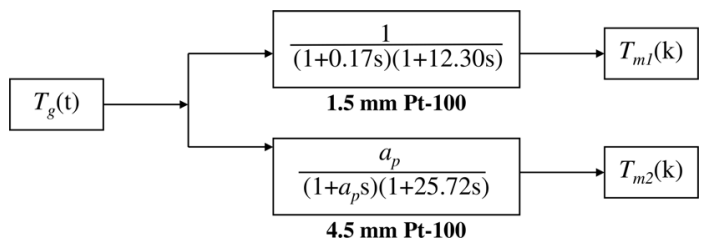

Fig. 10. Simulated Pt-100 output using second-order transfer functions.

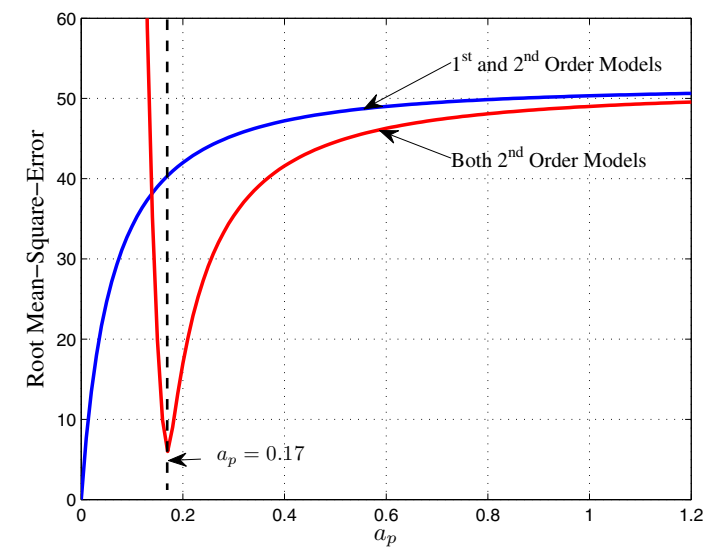

Fig. 11. Variation in mean-squared-error between $T_{g r}$ and $T_{g r e f}$ with $a_{p}$.

An interesting outcome of this study is that when the dominant poles of each transfer function are equal $\left(a_{p}=0.17\right)$, the reconstruction error approaches zero. With this in mind, the simulation was repeated. The second-order models in Tables I and II were again assumed, with the dominant pole in both transfer functions now being equal as in Fig. 12. Figure 13 shows that for this situation, the RMSE between simulated and reconstructed temperatures is reduced significantly.

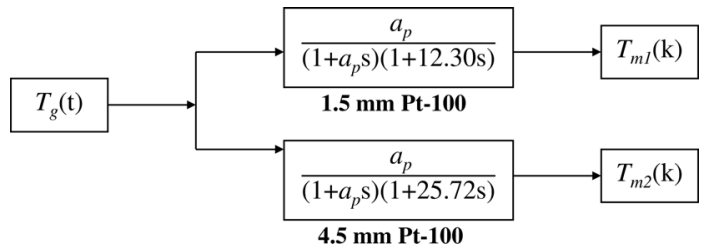

Fig. 12. Simulated Pt-100 output using second-order transfer functions.

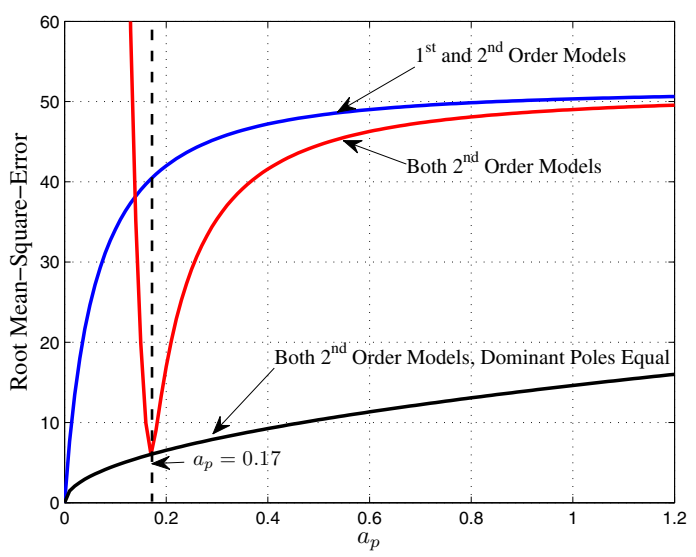

Fig. 13. RMSE between $T_{g r}$ and $T_{g r e f}$ against $a_{p}$.

To find out why this reduction in RMSE occurs when the dominant pole in each transfer function is equal, it is useful to more closely examine the mathematics behind each model. If a second-order transfer function of the form shown in Eq. (13) is factorised using partial fractions, the form shown in Eq. (14) follows. Equations (15) and (16) then represent Eq. (14) for two different Pt-100s.

$$
\begin{gathered}
G(s)=\frac{1}{\left(1+a_{p} s\right)\left(1+b_{p} s\right)} \\
G(s)=\frac{a_{p}}{\left(a_{p}-b_{p}\right)\left(1+a_{p} s\right)}+\frac{b_{p}}{\left(b_{p}-a_{p}\right)\left(1+b_{p} s\right)} \\
G_{1}(s)=\frac{a_{p 1}}{\left(a_{p 1}-b_{p 1}\right)\left(1+a_{p 1} s\right)}+\frac{b_{p 1}}{\left(b_{p 1}-a_{p 1}\right)\left(1+b_{p 1} s\right)} \\
G_{2}(s)=\frac{a_{p 2}}{\left(a_{p 2}-b_{p 2}\right)\left(1+a_{p 2} s\right)}+\frac{b_{p 2}}{\left(b_{p 2}-a_{p 2}\right)\left(1+b_{p 2} s\right)}
\end{gathered}
$$

As with the previous study, it was assumed that $a_{p 1}=$ $a_{p 2}=a_{p}$. Equations (15) and (16) can then be represented in the model shown in Fig. 14. The original second-order models are now effectively two first-order transfer functions that are pre-filtered by almost identical first-order ones. This then explains the reduced RMSE shown in Fig. 13. As $a_{p}$ increases, the amount of pre-filtering is increased, resulting in the loss of high frequency data, in turn resulting in increased errors between $T_{g r}$ and $T_{g r e f}$. Whilst this result is interesting, it 
would be extremely difficult to manufacture two Pt-100s with identical poles and so seems to be of little practical use.

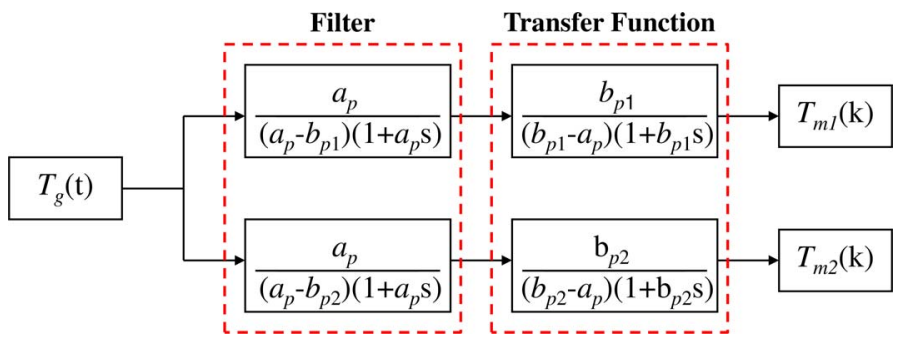

Fig. 14. Simulated Pt-100 output using second-order models with equal first poles.

It is essential that temperature measurement sensors to be used with dual-thermocouple characterisation techniques do not exhibit hysteresis effects. If this were the case, the sensor would have a path-dependent memory i.e. the output of the sensor could not be predicted without looking at the historical input before it reached its current value. Figures 15 and 16 show the response of a $1.5 \mathrm{~mm}$ wire-wound Pt-100 when subjected to a step increase and step decrease in gas temperature respectively. The time constant varies significantly between heating $\left(\tau_{h}=21.96 \mathrm{~s}\right)$ and cooling $\left(\tau_{c}=28.86 \mathrm{~s}\right)$, suggesting that $\mathrm{Pt}-100 \mathrm{~s}$ exhibit hysteresis. This is another obstacle preventing the use of Pt-100s with characterisation methods that assume first-order sensor response.

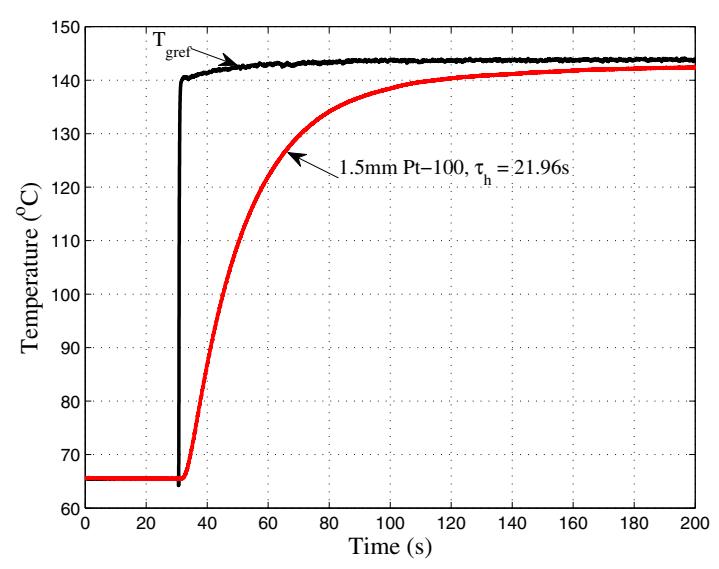

Fig. 15. Step increase in gas temperature measured using $1.5 \mathrm{~mm}$ Pt-100

\section{CONCLUSIONS}

1) Although it appeared that Pt-100s were relatively close to exhibiting first-order response, it was found that they could be more accurately modelled using a second-order model.

2) Analysis of experimental data showed that the inaccuracy of a first-model was such that temperature reconstructions were not possible through dual-thermocouple characterisation methods that assumed first-order response.

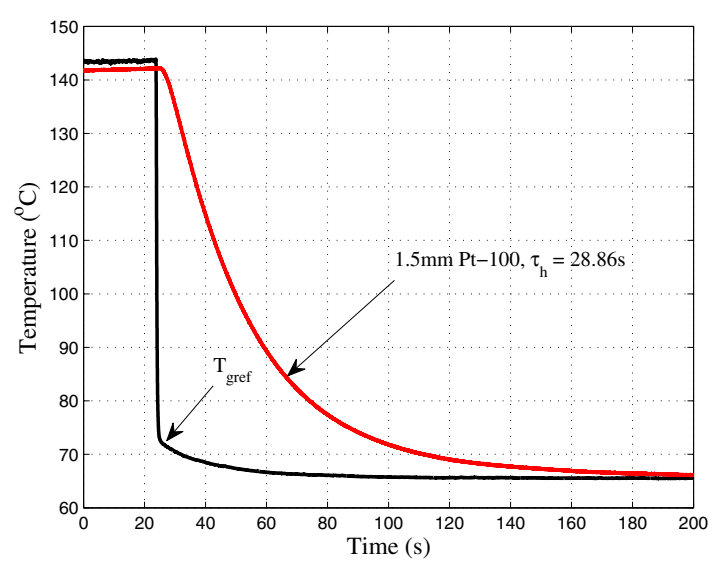

Fig. 16. Step decrease in gas temperature measured using $1.5 \mathrm{~mm}$ Pt-100

3) In simulation it was found that if a sensor exhibits even a marginally second-order response, it would produce highly inaccurate time constant estimates when a dualthermocouple characterisation scheme which assumes first-order is applied.

4) A further obstacle to the application of first-order modelling is the hysteresis effects exhibited by Pt-100s.

5) A higher-order model that more accurately reflects RTD response is necessary for successful dual-RTD characterisation.

6) Two sensors are required to identify the unknown parameters of a first-order sensor model; a higher-order model will require additional sensors if the unknown model parameters are to be found. Such a scheme would pose significant practical challenges.

\section{REFERENCES}

[1] T. Sidor, "Dynamic error correction of thin-wire resistance thermometers," J. Phys. E: Sci. Instrum., vol. 12, 1979.

[2] M. Tagawa and Y. Ohta, "Two-thermocouple probe for fluctuating temperature measurement in combustion: rational estimation of mean and fluctuating time constants," Combust. Flame, vol. 109, no. 4, pp. 549560, 1997.

[3] R. J. Kee, P. G. O'Reilly, P. T. McEntee, and R. Fleck, "Measurement of exhaust gas temperature in a high performance two-stroke engine," $S A E$ Trans., vol. 107, p. 983072, 1998.

[4] L. J. Forney and G. C. Fralick, "Two wire thermocouple: Frequency response in constant flow," Rev. Sci. Instrum., vol. 65, pp. 3252-3257, 1994.

[5] D. Bradley and K. J. Matthews, "Measurement of high gas temperatures with fine wire thermocouples," J. Mech. Eng. Sci., vol. 10, no. 4, pp. 299-305, 1968.

[6] T. Tsuji, Y. Nagano, and M. Tagawa, "Frequency response and instantaneous temperature profile of cold-wire sensors for fluid temperature fluctuation measurements," Exp. Fluids, vol. 13, no. 2-3, pp. 171-178, 1992.

[7] C. F. Hung, S. F. McLoone, R. Kee, and G. W. Irwin, "Difference equation approach to two-thermocouple sensor characterisation in constant velocity flow environments," Rev. Sci. Instrum., vol. 76, 2005.

[8] H. Pfriem, "Zur messung veränderlicher temperaturen von gasen und flüssigkeiten," Forschung im Ingenieurwesen, vol. 7, pp. 85-92, 1936.

[9] P. Cambray, "Measuring thermocouple time constants: a new method," Combustion Sci. Tech., vol. 45, pp. 221-224, 1986. 